\title{
User Clustering Scheme for Downlink of NOMA System
}

\author{
Li Li $^{1}$, Zhenghui Feng ${ }^{1}$, Yanzhi Tang ${ }^{1}$, Zhangjie Peng ${ }^{1,2 *}$, Lisen Wang ${ }^{1}$, Weilu Shao ${ }^{1}$ \\ 1 The College of Information, Mechanical and Electrical Engineering, Shanghai Normal University, \\ Shanghai 200234, China. \\ [e-mail: lilyxuan@shun.edu.cn, huifzsh@163.com, pengzhangjie@shnu.edu.cn] \\ 2 National Mobile Communications Research Laboratory, Southeast University, \\ Nanjing 210096, China. \\ *Corresponding author: Zhangjie Peng \\ Received July 11, 2019; revised October 15, 2019; accepted November 7, 2019; \\ published March 31, 2020
}

\begin{abstract}
An improved clustering scheme based on user group is proposed. Every two users are grouped among $N$-users in the allowed system according to their link gain from large to small. Each user group is numbered sequentially. Two user clusters are obtained according to the principle of maximizing link gain difference for the users in the first and last user groups. The remaining user groups are added to the two existing user clusters according to the parity of the group number. The clustering should be clustered again among the users in either user cluster if the throughput summation of a user cluster in NOMA is less than that of these users in orthogonal multiple access. The simulation results show that the proposed clustering scheme can increase the system throughput by about $8 \%$ compared with the hybrid clustering scheme when the number of users requiring service is 12 .
\end{abstract}

Keywords: NOMA; user clustering; power allocation

This work was supported in part by the NSFC under 61701307. And it was also supported in part by the open research fund of National Mobile Communications Research Laboratory, Southeast University (No. 2018D14). 


\section{Introduction}

The fifth generation (5G) wireless mobile communication has become a hot research area in recent years because of its high spectral efficiency, data rates and large capacity. Non-orthogonal Multiple Access (NOMA) is considered as one of the candidate access technologies for $5 \mathrm{G}$ wireless mobile communication because of its high spectral efficiency [1].

The NOMA system studied in this paper is based on the downlink of Long-Term Evolution (LTE) system. The interference between subchannels with orthogonal frequency division multiplexing (OFDM) multiple access scheme is ignored here. The NOMA system multiplexes the signals of multiple users in the power domain. Besides, the signals of users sharing the same subchannel are superimposed and transmitted over the channel. Channel models such as Rayleigh fading channels are described in the reference [2, 3]. Successive Interference Cancellation (SIC) receiver detects, reconstructs and cancels the non-orthogonal multiple access interference (MAI) at the receiver $[4,5]$.

In NOMA system, multiple users perform power multiplexing at the transmitting end and share the same subchannel, which form a user cluster. Therefore, the choice of user clustering [6] and power allocation [7] schemes will have a large impact on system performance. User pairing and power allocation are discussed in $[8,9]$. A greedy user selection and iterative sub-optimal power allocation algorithm based on Difference of Convex (DC) programming is presented in [8]. User pairing and power allocation(UPPA) based on proportional fairness is proposed in [9], but authors of [9] only considered 2-user NOMA system, more than 2 users in NOMA system had not been considered.

Various studies have shown that a reasonable user clustering scheme combined with optimal power allocation results will maximize system throughput. Literature [10] analyses the different impacts of user pairing on fixed power allocation NOMA (F-NOMA) system and cognitive-radio-inspired NOMA (CR-NOMA) system. According to the principle of maximizing the link gain difference, the user clustering scheme in [11] selects one user from each group to form a user cluster after grouping the users into 2,3,4 groups. Two users with the largest link gain are defined as the center users, two users with the least link gain are defined as the edge users, and other users are all the middle users. The clustering scheme[11] can easily group the middle users into the same user cluster, so the interference between these middle users is more serious, which is difficult to the interference elimination at the receiver. In reference [12], hybrid user clustering scheme is proposed to improve the throughput of edge users while resolving the problem of middle user interference in a cell, but the throughput of middle users needs to be improved. In reference [13], a slot-based user-clustering scheme is proposed to improve the throughput of users with similar link gain, but the reasonable resources allocation in time- and frequency-domain among users within the cluster is unsolved. The literature [14] proposed a vertical clustering scheme, which has higher requirements for the SIC receiver to correctly implement the SIC. In reference [15], hybrid multiple access scheme is adopted, orthogonal multiple access (OMA) is used for all cell central users, and then cell edge users are paired with each center user to realize NOMA. Since all paired users share the bandwidth of system, the problem of bandwidth allocation between user-pairs needs to be solved.

After the clustering procedure accomplished, the base station performs power allocation for each user. Power allocation is a crucial factor that affects the performance of power-domain NOMA system, which has attracted a lot of researchers' attentions [16-17]. Most of the 
existing research works mainly focus on fixed power allocation [18]. In [19], a low-complexity polynomial power allocation algorithm was investigated from a fairness standpoint. A power allocation method is proposed under the condition that the user's total rate is maximized according to the minimum user rate requirement [20, 21]. The difference between them is that fewer constraints are considered in [21] and the maximum number of users accommodated in a single cluster is obtained in [20]. However, the analytical expression of the upper limit of the number of users in a cluster remains to be studied. In addition, the joint subcarrier and power allocation problem was also studied in [22].

In order to decrease the serious interference between middle users[11] and to improve the throughput of middle users in hybrid clustering scheme [12], user clustering scheme is presented in this paper. The main contributions of this work are concluded as follows:

1) An improved user clustering scheme based on user group is proposed for downlink transmission of NOMA system. Each user group in this paper includes two users only, which is different from the grouping scheme in [11].

2) The power allocation results of each user in the cluster is obtained with the system throughput maximization as the objective function, total power, minimum transmission rate, and correct implementation of SIC as constraints.

3) The conditions for secondary clustering are proposed. When the number of system users increases, the effect of the clustering scheme proposed in this paper under quadratic clustering is verified.

The rest of this paper is organized as follows. System model for downlink of NOMA system is introduced in Section 2. The user clustering scheme is proposed in Section 3.1. Based on user clustering scheme, power allocation results are given in Section 3.2. Section 4 and Section 5 present the simulation results and conclusion.

\section{System Model}

Fig. 1 shows the NOMA downlink system model. Assuming there are $N$ users in a cell of NOMA cellular system. A center base station offers the possible service to the $N$ users. The system bandwidth $B_{s}$ is divided into $\Omega$ sub-channels with bandwidth $B$, it is assumed that $K$ users are non-orthogonal to the same sub-channel $(2 \leq K \leq N)$,

$P$ represents the total transmission power to each user cluster. For these $K$ users in cluster $i$, $s_{i, k}$ represents the message signal for the $k^{\text {th }}$ user in cluster $i$. Let $a_{i, k}$ represents the user power allocation coefficient of the $k^{\text {th }}$ user in cluster $i$. For cluster $i$, the superimposed signal sent by BS can be given by (1):

$$
x_{i}=\sum_{k=1}^{K} \sqrt{a_{i, k} P} s_{i, k}
$$

The signal received at the $k^{\text {th }}$ user in cluster $i$ is shown in (2)

$$
r_{i, k}=h_{i, k} X_{i}+n_{i, k}, k=1,2,3, \ldots \ldots, K,
$$

where the channel over each link is considered to be independent Rayleigh flat fading with channel coefficients $h_{i, k}, h_{i, k} \sim C N\left(0, d_{i, k}{ }^{-v}\right)$ represents the link gain of user $k$ in cluster $i[13] . d_{i, k}$ is the distance between the user $k$ and the central base station, $v$ is the path loss exponent in cluster $i . n_{i, k} \sim C N\left(0, \delta^{2}\right)$ is the additive white Gaussian noise with variance $\delta^{2}$. Formula (1) 
show that users sharing the same subchannel will receive the interference from other users in the same cluster. The $m^{\text {th }}$ user will have to detect and remove the data of all the $k^{\text {th }}$ users using SIC, while $m<k$.

Suppose $N$ users are divided into $I$ user clusters with $K$ users per cluster. The total system throughput can be expressed by[12]

$$
R_{\text {sum }}=\sum_{i=1}^{I}\left\{B \log _{2}\left(1+\rho_{i}\left|h_{i, 1}\right|^{2} a_{i, 1}\right)+\sum_{k=2}^{K} B \log _{2}\left(1+\frac{\rho_{i}\left|h_{i, k}\right|^{2} a_{i, k}}{1+\sum_{j=1}^{k-1} \rho_{i}\left|h_{i, k}\right|^{2} a_{i, j}}\right)\right\},
$$

where $\boldsymbol{\rho}_{\boldsymbol{i}}$ is the signal-to-noise ratio (SNR) to cluster $i$ in the base station. $h_{i, k}$ and $a_{i, k}$ are the link gain and the user power allocation coefficient of the $k^{\text {th }}$ user in cluster $i$. In formula (3), $B \log _{2}\left(1+\frac{\rho_{i}\left|h_{i, k}\right|^{2} a_{i, k}}{1+\sum_{j=1}^{k-1} \rho_{i}\left|h_{i, k}\right|^{2} a_{i, j}}\right)$ represents the throughput of the $k^{\text {th }}(\mathrm{k}>1)$ user; when $k=1$, the throughput expression is $B \log _{2}\left(1+\rho_{i}\left|h_{i, 1}\right|^{2} a_{i, 1}\right)$.

It can be seen from formula (3) that the total throughput of the NOMA system is related to the user clustering scheme.

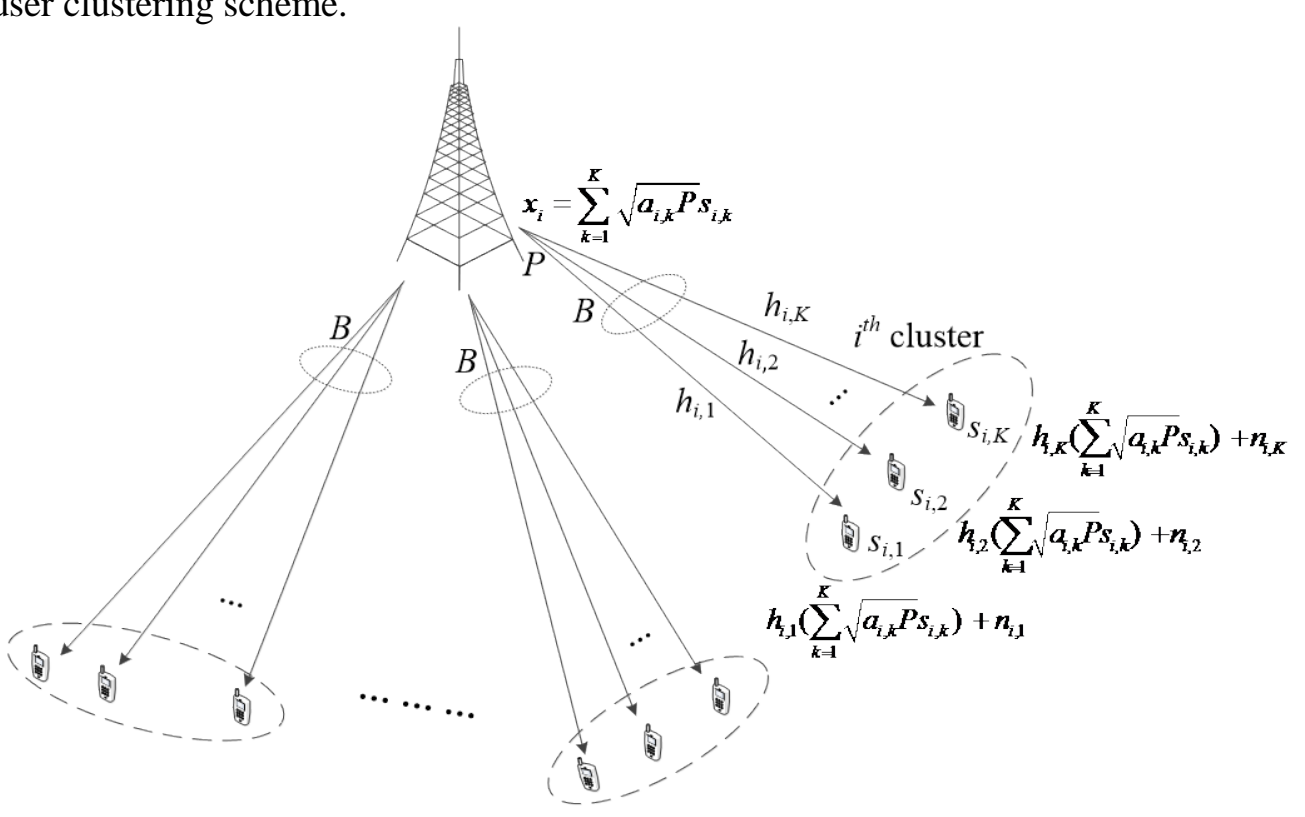

Fig. 1. NOMA downlink system model

\section{User Clustering in NOMA}

\subsection{Improved Clustering Scheme Based on User Groups}

An improved clustering scheme is proposed based on the throughput of NOMA system in order to improve the throughput of middle users. According to formula (3), for cluster $i$, the 
throughput of user $k$ increases with the increase of its own power allocation coefficient $a_{i, k}$ whereas decreases with the increase of other user's power allocation. So increase $a_{i, k}$ can improve throughput of user $k$. Based on this idea, the specific clustering scheme is as follows:

The users requiring downlink service are sorted by base station in descending order of their $\left|h_{n}\right|$ and denoted as $\mathrm{UE}_{n}(n=1,2,3, \ldots, N=I K)$, The sorted users are divided into $T=\lfloor N / 2\rfloor$ groups, $\lfloor\cdot\rfloor$ denotes rounding down. $G_{t}$ represents the $t^{\text {th }}$ user group

$$
G_{t}=\left\{\mathrm{UE}_{2 t-1}, \mathrm{UE}_{2 t}\right\}, t=1,2, \ldots \ldots, T
$$

The link gain difference maximization principle is adopted for the users in the first and the $T^{\text {th }}$ user groups to obtain two 2-user clusters $C_{1}=\left\{\mathrm{UE}_{1}, \mathrm{UE}_{2 T}\right\}, C_{2}=\left\{\mathrm{UE}_{2}, \mathrm{UE}_{2 T-1}\right\}[11]$.

For the $2^{\text {nd }}$ to the $(T-1)^{\text {th }}$ user groups, the users in the even group-index is added to $C_{1}$, the user in the odd group-index is added to $C_{2}$. For example, when the total number $T$ of user groups is even, the clustering result is

$$
\begin{aligned}
& C_{1}=\left\{\mathrm{UE}_{1}, G_{2}, G_{4}, G_{6}, \ldots \ldots, G_{T-2}, \mathrm{UE}_{2 T}\right\} \\
& C_{2}=\left\{\mathrm{UE}_{2}, G_{3}, G_{5}, G_{7}, \ldots \ldots, G_{T-1}, \mathrm{UE}_{2 T-1}\right\}
\end{aligned}
$$

When the number $T$ of user groups is odd, the clustering result is

$$
\begin{aligned}
& C_{1}=\left\{\mathrm{UE}_{1}, G_{2}, G_{4}, G_{6}, \ldots \ldots, G_{T-1}, \mathrm{UE}_{2 T}\right\} \\
& C_{2}=\left\{\mathrm{UE}_{2}, G_{3}, G_{5}, G_{7}, \ldots \ldots, G_{T-2}, \mathrm{UE}_{2 T-1}\right\}
\end{aligned}
$$

If the throughput summation of $K$ users in a single user cluster $C_{i}$ in the NOMA system is less than the throughput summation of these users in orthogonal multiple access, that is

$$
B \log _{2}\left(1+\rho_{i}\left|h_{i, 1}\right|^{2} a_{i, 1}\right)+\sum_{k=2}^{K} B \log _{2}\left(1+\frac{\rho_{i}\left|h_{i, k}\right|^{2} a_{i, k}}{1+\sum_{j=1}^{k-1} \rho_{i}\left|h_{i, k}\right|^{2} a_{i, j}}\right)<\frac{B}{K} \sum_{k=1}^{K} \log _{2}\left(1+\rho_{i}\left|h_{i, k}\right|^{2} b_{i, k}\right)
$$

In formula(7), $b_{i, k}$ is the power allocation factor of the $k^{\text {th }}$ user in the user cluster $C_{i}$ in the orthogonal multiple access mode. Then, the users in the user cluster $C_{i}$ need to be clustered again according to the above method until the sum of the throughputs of the users in the single user cluster is greater than the sum of the throughputs of the users in the orthogonal multiple access. After secondary clustering, $C_{1}$ will be divided into $C_{11}$ and $C_{12}$ while $C_{2}$ will be divided into $C_{21}$ and $C_{22}$, et. al. For example, based on formula(5), the secondary clustering result is:

$$
\begin{aligned}
& C_{11}=\left\{\mathrm{UE}_{1}, G_{2,2}, G_{4,1}, G_{6,2}, G_{8,1}, \mathrm{UE}_{2 T}\right\} \\
& C_{12}=\left\{G_{2,1}, G_{4,2}, G_{6,1}, G_{8,2}, G_{10,1}, G_{10,2}\right\} \\
& C_{21}=\left\{\mathrm{UE}_{1}, G_{3,2}, G_{5,1}, G_{7,2}, G_{9,1}, \mathrm{UE}_{2 T-1}\right\} \\
& C_{22}=\left\{G_{3,1}, G_{5,2}, G_{7,1}, G_{9,2}, G_{11,1}, G_{11,2}\right\}
\end{aligned}
$$

In formula(8), $G_{i, j}$ represents the $j^{\text {th }}$ user in user group $G_{i}$. The flow chart of user clustering is shown in Fig. 2. 


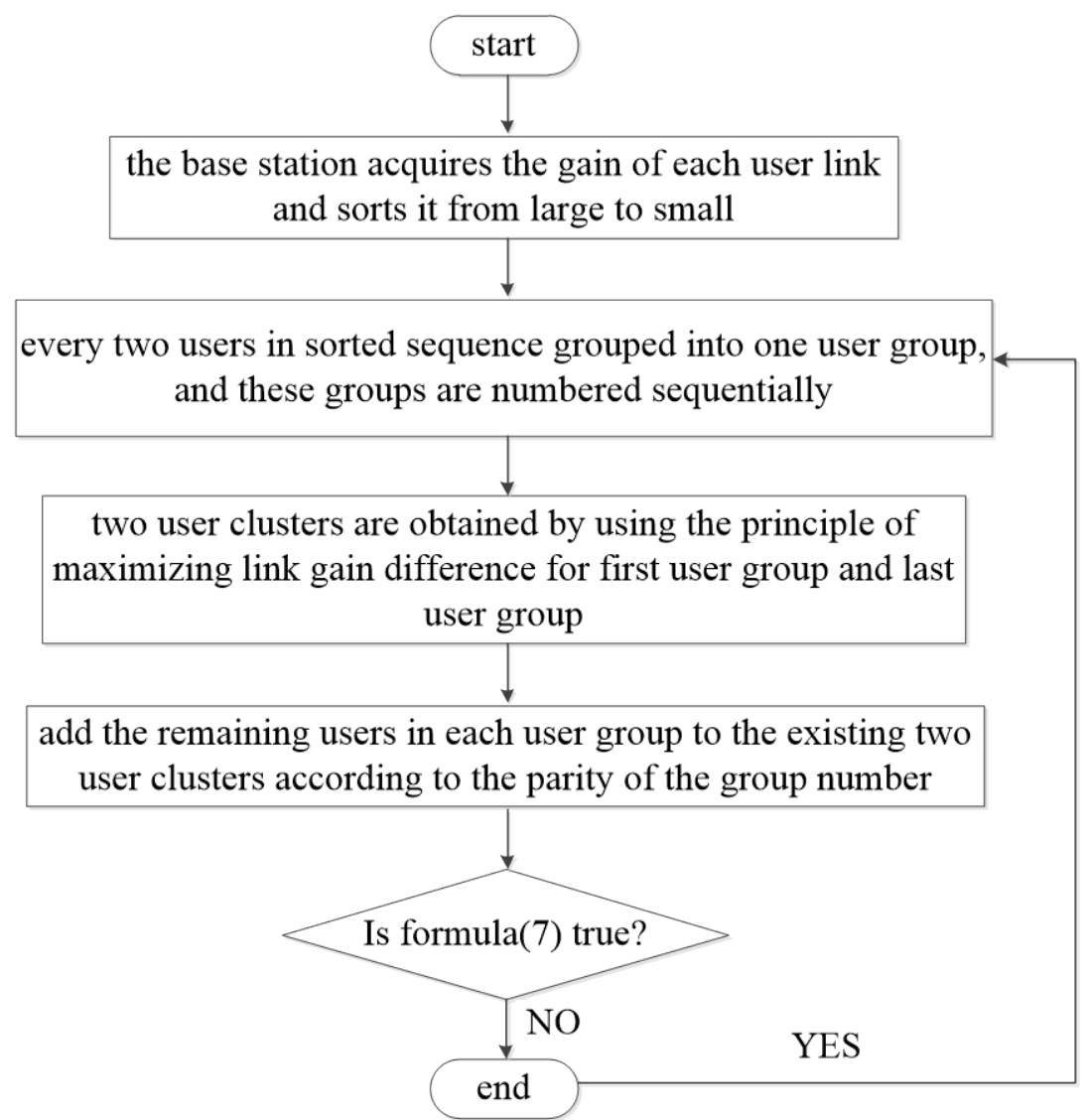

Fig. 2. Flow chart of user clustering

Since the system throughput performance is related to the results of the user power allocation within the cluster, the power allocation results between users in the cluster are given in section 3.2.

\subsection{Power Allocation of Users in a Cluster}

The throughput of the $K$-user cluster in the downlink of NOMA system is shown in formula (3). $R_{i, k}(k=1,2, \ldots \ldots, K)$ represents the minimum transmission rate of the $k^{\text {th }}$ user in cluster $i$, and $\boldsymbol{R}_{i, k}>\mathbf{0}$, then the power allocation problem of cluster $i$ can be further expressed as[11]:

$$
\max _{a_{i, k}} B \sum_{k=1}^{K} \log _{2}\left(1+\frac{\rho_{i}\left|h_{i, k}\right|^{2} a_{i, k}}{1+\sum_{j=1}^{k-1} \rho_{i}\left|h_{i, k}\right|^{2} a_{i, j}}\right)
$$

subject to:

$$
\sum_{k=1}^{K} a_{i, k} \leq 1
$$




$$
\begin{gathered}
B \log _{2}\left(1+\frac{\rho_{i}\left|h_{i, k}\right|^{2} a_{i, k}}{1+\sum_{j=1}^{k-1} \rho_{i}\left|h_{i, k}\right|^{2} a_{i, j}}\right) \geq R_{i, k}, \forall k=1,2, \ldots \ldots, K \\
\rho_{i}\left|h_{i, k-1}\right|^{2} a_{i, k}-\sum_{j=1}^{k-1} \rho_{i}\left|h_{i, k-1}\right|^{2} a_{i, j} \geq P_{t o l}, \forall k=2,3, \ldots \ldots, K
\end{gathered}
$$

$P_{\text {tol }}$ refers to the minimum power difference between the signal to be decoded and the remaining undecoded signal. Formula (10) represents the constraint of the sum of the power allocation coefficient of the NOMA system, formula (11) denotes the minimum transmission rate constraint, and formula (12) represents SIC constraints.

According to formulas (9) to (12), the Lagrange objective function is constructed as follows:

$$
\begin{aligned}
L\left(a_{i}, \mu_{i}, v_{i}, \lambda_{i}\right)= & B \sum_{k=1}^{K} \log _{2}\left(1+\frac{\rho_{i}\left|h_{i, k}\right|^{2} a_{i, k}}{1+\sum_{j=1}^{k-1} \rho_{i}\left|h_{i, k}\right|^{2} a_{i, j}}\right)+\mu_{i}\left(1-\sum_{k=1}^{K} a_{i, k}\right) \\
& +\sum_{k=1}^{K} v_{i, k}\left\{\rho_{i}\left|h_{i, k}\right|^{2} a_{i, k}-\left(1+\sum_{j=1}^{k-1} \rho_{i}\left|h_{i, k}\right|^{2} a_{i, j}\right) \times\left(\varphi_{i, k}-1\right)\right\}+\sum_{k=2}^{K} \lambda_{i, k}\left(\rho_{i}\left|h_{i, k-1}\right|^{2} a_{i, k}-\sum_{j=1}^{k-1} \rho_{i}\left|h_{i, k-1}\right|^{2} a_{i, j}-P_{t o l}\right)
\end{aligned}
$$

Among them, $a_{i} \equiv\left\{a_{i, 1}, a_{i, 2}, \ldots \ldots, a_{i, K}\right\}$ is power allocation coefficient combination in cluster $i ; \quad v_{i} \equiv\left\{v_{i, 1}, v_{i, 2}, \ldots \ldots, v_{i, K}\right\} \quad, \quad \lambda_{i} \equiv\left\{\lambda_{i, 2}, \lambda_{i, 3}, \ldots \ldots, \lambda_{i, K}\right\} \quad$ are Lagrange coefficient combinations, and $\varphi_{i, k}=2^{R_{i, k} / B}$. The Lagrange multiplier method is used to optimize the objective function to obtain the optimal power allocation. We can write KKT(Karush-Kuhn-Tucher) conditions as follows:

$$
\begin{aligned}
\frac{\partial L}{\partial a_{i, k}}= & \frac{\rho_{i}\left|h_{i, k}\right|^{2}}{\ln 2\left(1+\sum_{j=1}^{k} \rho_{i}\left|h_{i, k}\right|^{2} a_{i, j}\right)}-\sum_{u=k+1}^{K} \frac{\rho_{i}^{2}\left|h_{i, u}\right|^{4} a_{i, u}}{\ln 2\left[\left(1+\sum_{j=1}^{u} \rho_{i}\left|h_{i, u}\right|^{2} a_{i, j}\right)\left(1+\sum_{j=1}^{u-1} \rho_{i}\left|h_{i, u}\right|^{2} a_{i, j}\right)\right]}-\mu_{i}+v_{i, k} \rho_{i}\left|h_{i, k}\right|^{2} \\
& -\sum_{u=k+1}^{K}\left(\varphi_{i, u}-1\right) v_{i, u} \rho_{i}\left|h_{i, u}\right|^{2}+\lambda_{i, k} \rho_{i}\left|h_{i, k-1}\right|^{2}-\sum_{j=k+1}^{K} \lambda_{i, j} \rho_{i}\left|h_{i, j-1}\right|^{2}=0, \forall k=1,2, \ldots, K \\
\frac{\partial L}{\partial \mu_{i}}= & 1-\sum_{k=1}^{K} a_{i, k}=0, \text { if } \mu_{i} \geq 0 \\
\frac{\partial L}{\partial v_{i, k}}= & \rho_{i}\left|h_{i, k}\right|^{2} a_{i, k}-\left(1+\sum_{j=1}^{k-1} \rho_{i}\left|h_{i, k}\right|^{2} a_{i, j}\right) \times\left(\varphi_{i, k}-1\right)=0, \text { if } v_{i, k} \geq 0, \forall k=1,2, \ldots, K \\
\frac{\partial L}{\partial \lambda_{i, k}}= & \rho_{i}\left|h_{i, k-1}\right|^{2} a_{i, k}-\sum_{j=1}^{k-1} \rho_{i}\left|h_{i, k-1}\right|^{2} a_{i, j}-P_{t o l}=0, \text { if } \lambda_{i, k} \geq 0, \forall k=2, \ldots, K
\end{aligned}
$$

The combinations of Lagrange coefficients satisfying the KKT condition are obtained through formula (14) to formula (17) and then choosing one combination to solve the power distribution factor[20]. In this paper, when the coefficients are combined into $\left\{\mu_{i}, \lambda_{i, 2}, \lambda_{i, 3}, \ldots\right.$, $\left.\lambda_{i, K}\right\}$, the power allocation results of each user in cluster $i$ are shown in (18)[20]. 


$$
\begin{aligned}
& a_{i, 1}=\frac{1}{2^{K-1}}-\frac{P_{t o l}}{2\left|h_{i, 1}\right|^{2} \rho_{i}}-\cdots \cdots-\frac{P_{t o l}}{2^{K-1}\left|h_{i, K-1}\right|^{2} \rho_{i}} \\
& a_{i, k}=\frac{1}{2^{K-k+1}}+\frac{P_{t o l}}{2\left|h_{i, k-1}\right|^{2} \rho_{i}}-\frac{P_{t o l}}{2^{2}\left|h_{i, k}\right|^{2} \rho_{i}}-\frac{P_{t o l}}{2^{3}\left|h_{i, k+1}\right|^{2} \rho_{i}}-\cdots \cdots-\frac{P_{t o l}}{2^{K-k+1}\left|h_{i, K-1}\right|^{2} \rho_{i}}, k=2,3, \ldots . ., K
\end{aligned}
$$

\section{Numerical Results and Discussions}

\subsection{Parameter settings of NOMA downlink}

For the scenario in Fig. 1, assume the number $N$ of system users is 12 and system sub-channel bandwidth $B$ is $180 \mathrm{kHz}$.

The distance $d_{i, k}$ between the user $k$ in cluster $i$ and the central base station (CBS) is assumed normalized by the radius of CBS coverage; $d_{i, k}$ varies between 0 and 1 as users moves randomly in the cell. The link gain $h_{i, k} \sim \operatorname{CN}\left(0, d_{i, k}^{-4}\right)$, The detection threshold $P_{\text {tol }}$ at the SIC receiver in formula (12) is $10 \mathrm{dBm}$. Based on the above assumptions, MATLAB is used to simulate the throughput under different clustering schemes.

Fig. 3 shows the user clustering results when the number of system users is $12(T=6)$, Users in the two user clusters are $C_{1}=\left\{\mathrm{UE}_{1}, \mathrm{UE}_{3}, \mathrm{UE}_{4}, \mathrm{UE}_{7}, \mathrm{UE}_{8}, \mathrm{UE}_{12}\right\}$ and $C_{2}=\left\{\mathrm{UE}_{2}, \mathrm{UE}_{5}, \mathrm{UE}_{6}\right.$, $\left.\mathrm{UE}_{9}, \mathrm{UE}_{10}, \mathrm{UE}_{11}\right\}$ respectively. If the hybrid clustering scheme is adopted when the number of user groups increases to 6 , users in the two clusters are $C_{1}{ }^{\prime}=\left\{\mathrm{UE}_{1}, \mathrm{UE}_{3}, \mathrm{UE} 5, \mathrm{UE}_{7}, \mathrm{UE}_{10}, \mathrm{UE}_{12}\right\}$ and $C_{2}=\left\{\mathrm{UE}_{2}, \mathrm{UE}_{4}, \mathrm{UE}_{6}, \mathrm{UE}_{8}, \mathrm{UE}_{9}, \mathrm{UE}_{11}\right\}$ respectively.

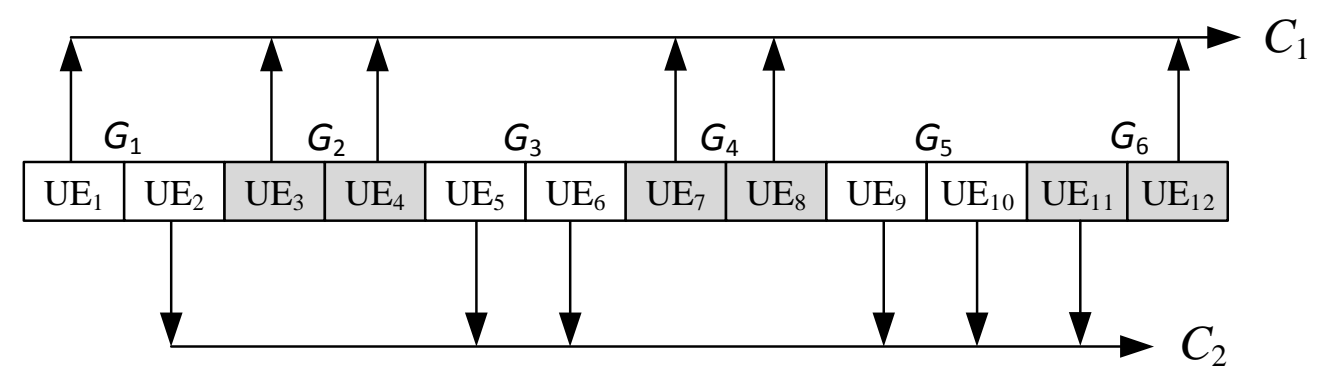

Fig. 3. Example to the proposed group-based user clustering scheme

\subsection{Simulation results discussion}

As the positions of middle user 4 , user 8 , user 5 , and user 9 in clustering results $C_{1}, C_{2}$ are different comparing with their positions in $C_{1}$ ', $C_{2}$ ', these middle users' throughput will be different according to the principle of power domain NOMA.

Fig. 4 shows the throughput of middle user 4 and user 8 is larger for the proposed clustering scheme than that of the hybrid clustering scheme. Whereas Fig. 5 shows the throughputs of middle user 5 and user 9 is smaller for the proposed clustering scheme than that of hybrid clustering scheme. 


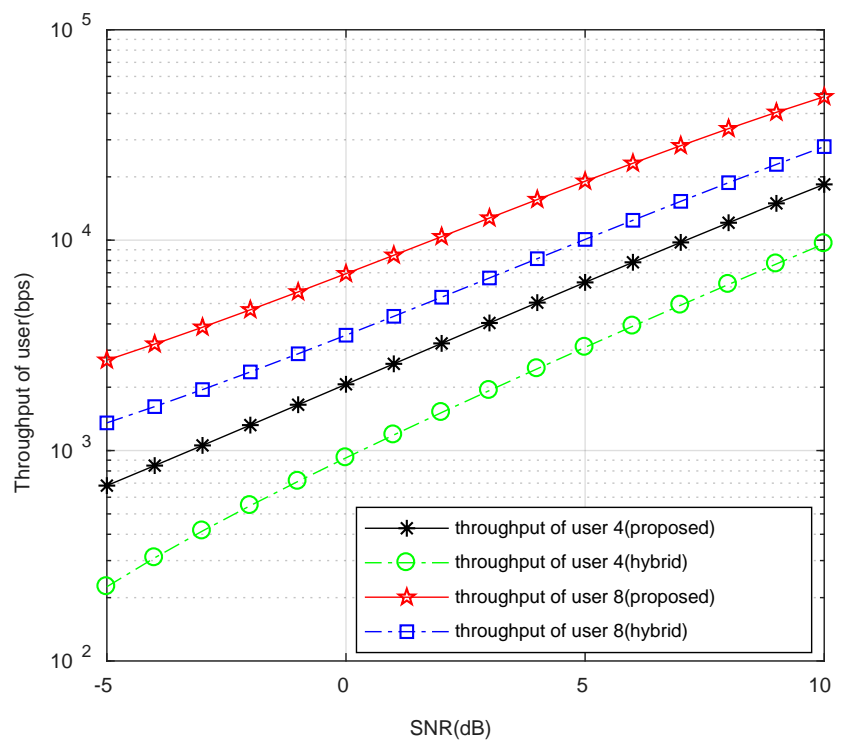

Fig. 4. Throughput of user 4 and user 8 under two different clustering schemes $(N=12)$

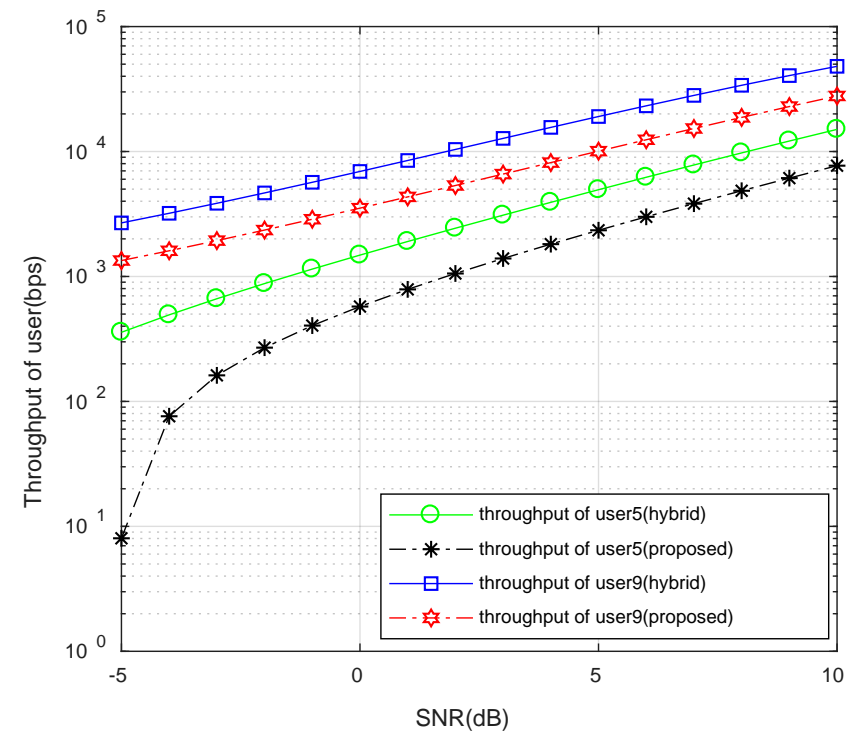

Fig. 5. Throughput of user 5 and user 9 under two different clustering schemes $(N=12)$

How is about the total system throughput in the downlink NOMA system then? To address this question, the throughput difference of user $n$ with the above two clustering schemes is defined as $\Delta t h_{n}$ showing in formula (19).

$$
\Delta t h_{n}=T h_{n}^{\text {proposed }}-T h_{n}^{\text {hybrid }}, n=1,2, \ldots \ldots, N \text {, }
$$


where $T h_{n}^{\text {proposed }}, T h_{n}^{\text {hybrid }}$ represent the throughputs of user $n$ with the proposed clustering scheme and that of with hybrid clustering scheme.

The growth rate $G_{t h}$ of total system throughput is shown in formula (20).

$$
G_{t h}=\frac{\sum_{n=1}^{N} \Delta t h_{n}}{R_{\text {sum }}^{\text {hybrid }}}
$$

$R_{\text {sum }}^{\text {hybrid }}=\sum_{n=1}^{N} T h_{n}^{\text {hybrid }}$ represents the total system throughput with hybrid clustering scheme.

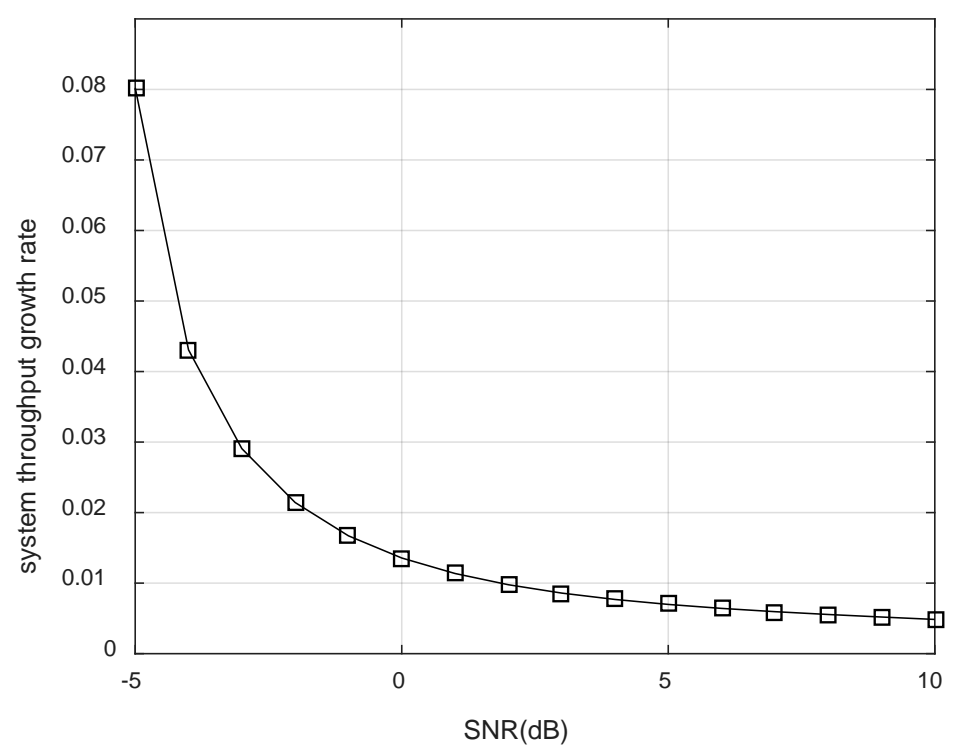

Fig. 6. System throughput growth rate compared with hybrid clustering scheme $(N=12)$

Fig. 6 shows the growth rate $G_{t h}$ of total system throughput of the proposed clustering scheme is $8 \%$ greater than that of the hybrid clustering scheme when SNR is $-5 \mathrm{~dB}$. Besides, the growth rate $G_{t h}$ of total system throughput decreases with the increase of SNR.

As the number of system users increases, the throughput of user clusters after once clustering gradually decreases, even less than the throughput of the user cluster under OMA. In this case, for example, if the number of system users increases to 24 , after the proposed clustering scheme proceeded once, the throughput of the resulting two cluster is no better than that of using hybrid clustering scheme. In order to improve the throughput better in this case, a secondary clustering procedure for each cluster with the proposed clustering scheme is suggested. The result after the secondary clustering is $C_{11}=\{1,4,7,12,15,24\}, C_{12}=$ $\{3,8,11,16,19,20\}, \quad C_{21}=\{2,6,9,14,17,23\}, \quad C_{22}=\{5,10,13,18,21,22\}$. For comparing convenience, the clustering result based on the idea of hybrid clustering scheme is $\{1,5,9,13,20,24\},\{2,6,10,14,19,23\},\{3,7,11,15,18,22\}$ and $\{4,8,12,16,17,21\}$ when the number of user groups increases to 4 . 


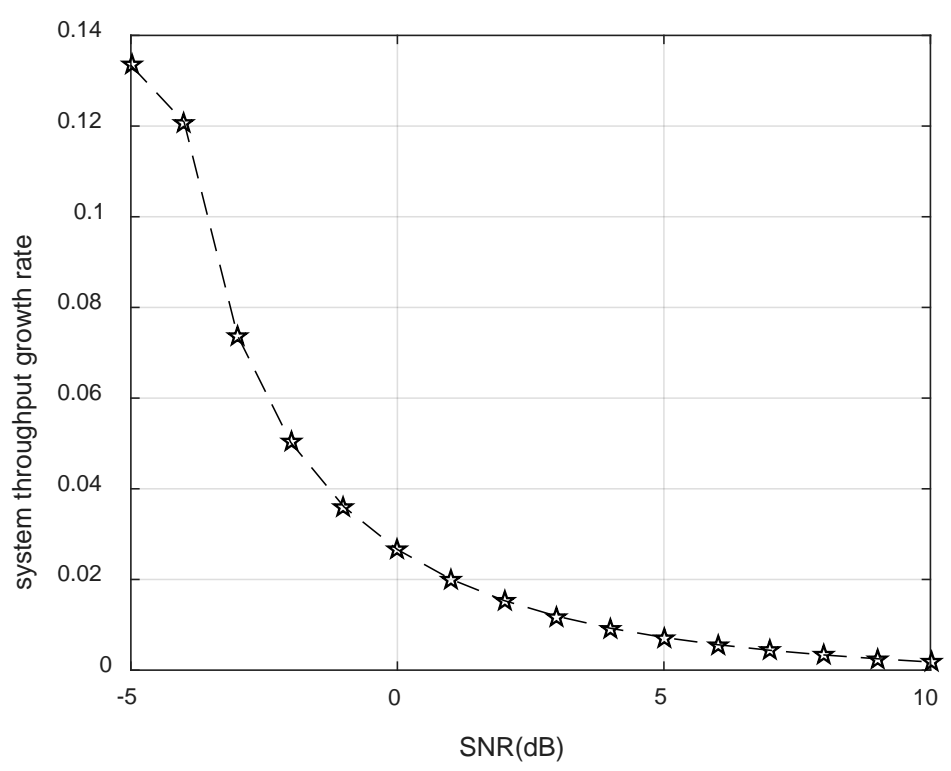

Fig. 7. System throughput growth rate compared with hybrid clustering scheme after secondary clustering $(N=24)$

Fig. 7 shows the total throughput growth rate of the system after secondary clustering is $13 \%$ greater than the hybrid clustering scheme when SNR is $-5 \mathrm{~dB}$, which demonstrates the system throughput of the proposed clustering scheme is higher than that of the hybrid clustering scheme. This is because, after secondary clustering with the proposed scheme, the interference between users in each cluster reduced, resulting in an increase in throughput.

\section{Conclusion}

The user clustering problem of NOMA in 5G mobile communication is studied in this paper. An improved clustering scheme based on user group is proposed, which can improve further the total throughput of the NOMA downlink system by increasing some middle users' throughput. The simulation results show that the clustering scheme proposed in this paper effectively improves the throughput of some middle users compared with hybrid clustering scheme. The system throughput has increased comparing with that of the hybrid clustering method. When the number of system users increased, a secondary clustering procedure might be need to obtain a system throughput improvement desired. However, the number of users that can be accommodated in a single user cluster and how many user clusters need to be clustered at most needs to be further studied.

\section{References}

[1] Yingmin Wang, Bin Ren, Shaohui Sun, et al., "Analysis of Non-Orthogonal Multiple Access for 5G," China Communications, 13(2), 52-56, 2016. Article (CrossRef Link)

[2] J. Chen, X. Ge, Q. Ni, “Coverage and Handoff Analysis of 5G Fractal Small Cell Networks," IEEE Transactions on Wireless Communications, 18(2), 1263-1276, 2019. Article (CrossRef Link)

[3] Y Jiang, X. Ge, Y Zhong, et al., "A New Small-World IoT Routing Mechanism based on Cayley Graphs,” IEEE Internet of Things Journal, 1-12, 2019.Article (CrossRef Link) 
[4] ISLAM S M R, AVAZOV N, DOBRE O A, "Power-domain non-orthogonal multiple access (NOMA) in 5G systems: potentials and challenges,” IEEE Communications Surveys \& Tutorials, 19(2), 721-742, 2016. Article (CrossRef Link)

[5] N. Otao, Y. Kishiyama, and K. Higuchi, "Performance of non-orthogonal access with SIC in cellular downlink using proportional fair-based resource allocation,” IEICE Transactions on Communications, 98(2), 344-351, 2015. Article (CrossRef Link)

[6] ZHANG H, ZHANG D, MENG W, et al., "User pairing algorithm with SIC in non-orthogonal multiple access system,” in Proc. of IEEE International Conference on Communications (ICC), 1-6, 2016. Article (CrossRef Link)

[7] XU W, LI X, LEE C, et al., “Joint Sensing Duration Adaptation, User Matching, and Power Allocation for Cognitive OFDM-NOMA Systems," IEEE Transactions on Wireless Communications, 17(2), 1269-1282, 2018. Article (CrossRef Link)

[8] LIU F, MÄHÖNEN P, PETROVA M, "Proportional fairness-based user pairing and power allocation for non-orthogonal multiple access,” in Proc. of IEEE 26th Annual International Symposium on Personal, Indoor, and Mobile Radio Communications (PIMRC), 1127-1131, 2015. Article (CrossRef Link)

[9] PARIDA P, DAS S S, "Power allocation in OFDM based NOMA systems: A DC programming approach,” in Proc. of IEEE Globecom Workshops (GC Wkshps), 1026-1031, 2014. Article (CrossRef Link)

[10] Ding Z, Fan P, Poor V, "Impact of user pairing on 5G non-orthogonal multiple access downlink transmissions,” IEEE Transactions on Vehicular Technology, 65(8), 6010-6023, 2015. Article (CrossRef Link)

[11] M. S. Ali, H. Tabassum, E. Hossain, "Dynamic user clustering and power allocation for uplink and downlink non-orthogonal multiple access (NOMA) systems,” IEEE Access, 4, 6325-6343, 2016. Article (CrossRef Link)

[12] M. B. Shahab, M. Irfan, M. F. Kader, et al., "User pairing schemes for capacity maximization in non-orthogonal multiple access systems," Wireless Communications and Mobile Computing, 16(17), 2884-2894, 2016. Article (CrossRef Link)

[13] M. B. Shahab, Soo Young Shin, "A Time Sharing Based Approach to Accommodate Similar Gain Users in NOMA for 5G Networks," in Proc. of IEEE 42nd Conference on Local Computer Networks Workshops (LCN Workshops), 142-147, 2017. Article (CrossRef Link)

[14] Z.Q. Al-Abbasi D.K.C. So, "User-Pairing Based Non-Orthogonal Multiple maAccess (NOMA) System,” in Proc. of IEEE Vehicular Technology Conference (VTC Spring), 1-5, 2016. Article (CrossRef Link)

[15] Dehuan Wan, Miaowen Wen, Yun Liu, et al., "User Pairing Strategy: A Novel Scheme for Non-Orthogonal Multiple Access Systems," in Proc. of IEEE Globecom Workshops (GC Wkshps), 1-6, 2017. Article (CrossRef Link)

[16] Z. Yang, Z. Ding, P. Fan, and N. Al-Dhahir, “A general power allocation scheme to guarantee quality of service in downlink and uplink NOMA systems," IEEE Transactions on Wireless Communications, 15(11), 7244-7257, 2016. Article (CrossRef Link)

[17] L. Lei, D. Yuan, C. K. Ho, and S. Sun, "Power and channel allocation for non-orthogonal multiple access in 5G systems: Tractability and computation," IEEE Transactions on Wireless Communications, 15(12), 8580-8594, 2016. Article (CrossRef Link)

[18] Z. Ding, Z. Yang, P. Fan, and H. V. Poor, "On the performance of non-orthogonal multiple access in 5G systems with randomly deployed users," IEEE Signal Processing Letters, 21(12), 1501-1505, 2014. Article (CrossRef Link)

[19] S. Timotheou and I. Krikidis, "Fairness for non-orthogonal multiple access in 5G systems," IEEE Signal Processing Letters, 22(10), 1647-1651, 2015. Article (CrossRef Link)

[20] FENG Z H, LI L, LIN Z, et al., "Research on maximum number of users in downlink cluster of non-orthogonal multiple access system," Journal of Shanghai Normal University (Natural Sciences), 48(1), 5-6, 2019. Article (CrossRef Link) 
[21] Zhaohui Yang, Xu Wei, Cunhua Pan, Yijin Pan, and Chen Ming, “On the optimality of power allocation for NOMA downlinks with individual Qos constraints,” IEEE Communications Letters, 21(7), 1649-1652, 2017. Article (CrossRef Link)

[22] L. Song, Y. Li, Z. Ding, and H. V. Poor, "Resource management in non-orthogonal multiple access systems: State of the art and research challenges,” arXiv:1610.09465, pp. 1-14, 2017. Article (CrossRef Link)
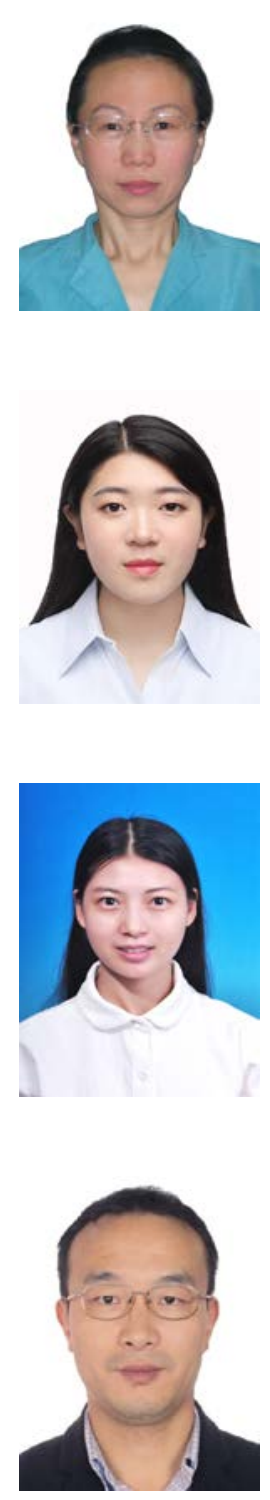

Li Li: She received the B.S. EE degree from Tsinghua University in 1985, the M.S. EE degree from China Research Institute of Radiowave Propagation in 1988, and Ph.D degree in science from Peking University in 1997. Her research interest is currently at the adaptive digital signal processing, spectrum allocation and interference alignment algorithms in heterogeneous cognitive radio network; network modeling of ultra dense wireless mobile communication.

Zhenghui Feng: She is currently pursuing a master degree at the College of Information, Mechanical and Electrical Engineering in Shanghai Normal University, Shanghai, China. Her research interests include wireless mobile communication and adaptive singal processing.

Yanzhi Tang: She is currently pursuing a master degree at the College of Information, Mechanical and Electrical Engineering in Shanghai Normal University, Shanghai, China. Her current research interests is wireless sensor network and wireless communication .

Zhangjie Peng: He received the B.S. degree from Southwest Jiaotong University, Chengdu, China, in 2004, and the M.S. and Ph.D. degrees from Southeast University, Southeast University, Nanjing, China, in 2007, and 2016, respectively, all in Communication and Information Engineering. He is currently an Associate Professor at the College of Information, Mechanical and Electrical Engineering, Shanghai Normal University, Shanghai 200234, China. His research interests include cooperative communications, information theory, physical layer security, and machine learning for wireless communications. 


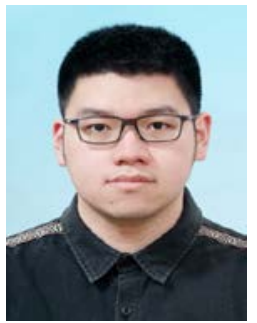

Lisen Wang: He is currently pursuing a master degree at the College of Information, Mechanical and Electrical Engineering in Shanghai Normal University, Shanghai, China. His research interests is currently at the adaptive singal processing and wireless communication.

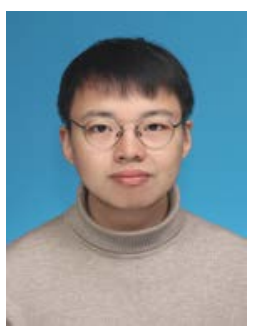

Weilu Shao: He received the Bachelor degree from Shanghai Normal University in 2017. He is currently pursuing a master degree at Shanghai Normal University. His research interest is currently at the improvement of MAC protocol in mobile ad hoc network. 